\title{
Magnetic-ordered and superconducting units inside surface layers of PbTe:Eu crystals grown from melt by using the Bridgman method
}

\author{
D.M. Zayachuk ${ }^{1}$, O.S. Ilyina ${ }^{1}$, D. Kaczorowski ${ }^{2}$, V.I. Mikityuk ${ }^{3}$, and V.V. Shlemkevych ${ }^{3}$ \\ ${ }^{1}$ Lviv Polytechnic National University, 12, Bandera str., 79013 Lviv, Ukraine \\ Phone: +38 (032) 258-21-13; fax: +38 (032) 258-26-80; e-mail: zayachuk@polynet.lviv.ua \\ ${ }^{2}$ Institute of Low Temperature and Structure Research, Polish Academy of Sciences, \\ 50-950 Wroclaw 2, P. O. Box 1410, Poland \\ ${ }^{3}$ Yuri Fedkovich Chernivtsy National University, \\ 2, Kotsyubynskoho str., 58012 Chernivtsy, Ukraine
}

\begin{abstract}
Presented in this work are the results of the first systematic study of magnetic properties inherent to surface layers of PbTe:Eu crystals grown from melt with a low initial concentration of Eu impurity $N_{\mathrm{Eu}}^{\text {int }}\left(\mathrm{ml}\right.$ ) (about $1 \cdot 10^{19} \mathrm{~cm}^{-3}$ ) using the Bridgman method. The magnetic field dependences of magnetization at the temperature $1.72 \mathrm{~K}$ and temperature dependences of magnetic susceptibility within the temperature range 1.7 to $10 \mathrm{~K}$ of the surface powder samples has been investigated. Inside the surface layers, formation of both small magnetic complexes based on $\mathrm{Eu}$ impurity and lead-based inclusions superconducting at low temperatures, which originate in a correlated way during the growth process of doped ingots, has been established. The observed correlations are manifested in a consistent distribution of both europium-based and leadbased units along the lateral surface, where the probability of their formation increases towards the end of the doped ingot. Based on the analysis of the obtained magnetic data, it has been suggested that the lead-based inclusions, being formed within the surface layer of PbTe:Eu crystals during the process of their growth and passing into a superconducting state on cooling, are the type II superconductors with a very high top critical field $H_{c 2}$ at low temperatures. At $T=1.7 \mathrm{~K}, H_{c 2}$ of the inclusions is estimated to exceed $50.0 \mathrm{kOe}$. The important role of the background oxygen impurity in formation of magnetic properties of the surface layers of $\mathrm{PbTe}: \mathrm{Eu}$ crystals has been suggested.
\end{abstract}

Keywords: IV-VI crystals, surface, rare earth impurities, magnetization, magnetic susceptibility, magnetic complexes, superconducting inclusions.

Manuscript received 17.07.13; revised version received 11.09.13; accepted for publication 23.10.13; published online 16.12.13.

\section{Introduction}

Unique possibilities for application of the impurities of rare earth elements (REE) to influence the properties of semiconductor crystal structures are well known. They are a powerful technological tool for cleaning semiconductor crystals and thin films of background uncontrolled impurities $[1,2]$. Their use allows to create the diluted magnetic solid solutions based on different semiconductors, which, in turn, allows to efficiently control physical characteristics and parameters of these materials using an external magnetic field [3-6]. REE impurities are used in practice for manufacturing semimagnetic semiconductor superlattices and other devices based on semiconductor heterostructures $[7,8]$.

Our recent studies [9] have revealed another unique feature in behaviour of REE impurities, which can be related to the well-known effect of small doses. On the example of Eu impurity in PbTe crystals it has been shown that if the PbTe:Eu crystal is grown by the Bridgman method from melt with very low initial concentrations of $\mathrm{Eu}$ impurity $\left(10^{19} \mathrm{~cm}^{-3}\right.$ and less $)$, it is 
possible to form a specific two-section crystal with a doped surface and undoped volume. In our technological experiments, using crystal ingots with the diameter $10 \mathrm{~mm}$, the thickness of the doped surface layer was up to ten micrometers, namely, several deciles of the percent of the crystal radius. We also showed that, within these surface layers inclusions, the passage into a superconducting state can take place on cooling [10]. It has been concluded that these inclusions are most likely $\mathrm{Pb}$-based. The goal of this study is to establish regularities of formation of the mentioned inclusions within the surface layers of PbTe:Eu crystals grown using the Bridgman method from melts doped with a low initial concentration of impurity, and search for correlations of their behavior with that of the Eu doping impurity.

\section{Samples and experiment}

The ingot chosen for investigation was grown using the Bridgman method from melt and had the initial concentration of $\mathrm{Eu}$ impurity about $1 \cdot 10^{19} \mathrm{~cm}^{-3}$. The distribution of $\mathrm{Eu}$ impurity was investigated in detail by using the methods of X-ray fluorescent element analysis and Secondary Neutral Mass Spectrometry (ingot 2 from [9]). So, we can be sure that the whole doping impurity is located within the ingot surface layers having the thickness not larger than $20 \mu \mathrm{m}$. The investigations of the surface layers were performed measuring magnetization and magnetic susceptibility (MS). For this purpose, as thin as possible surface layers were removed mechanically from the different part of the lateral surface and triturated. Three samples were prepared from these powders as specified in Table. One extra sample was fabricated out of the initial 3-mm long conical part of the ingot.

Magnetic measurements were performed at low temperatures within the range 1.7 to $10 \mathrm{~K}$ and under applied magnetic fields up to $50 \mathrm{kOe}$ using a Quantum Design MPMS-5 superconducting quantum interference device (SQUID) magnetometer.

\section{Experimental results}

The experimental data of the investigations of both magnetic field dependences of magnetization $M(H)$ at the temperature $1.72 \mathrm{~K}$ and temperature dependences of MS $\chi(T)$ at the magnetic field 300 Oe of the surface powder samples are presented in Figs 1 and 2 .

The following results are the most important to understand the regularities of formation of the surface layers of $\mathrm{PbTe}: \mathrm{Eu}$ ingots grown from melt doped with a low Eu impurity concentration.

(i) Only for the sample fabricated from the beginning of the conical part of the ingot (the sample 1), magnetization under the change of magnetic field up and down remains the same over the whole range of the investigated fields.

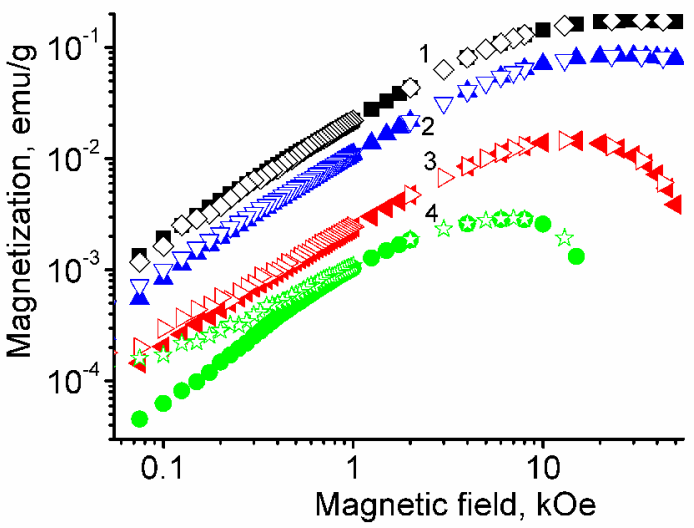

Fig. 1. Magnetization of the investigated samples vs. magnetic field at the temperature $1.72 \mathrm{~K}$. The solid symbols correspond to the increasing magnetic field; the open ones correspond to the decreasing magnetic field. The indexes of the curves correspond to the sample indexes from Table.

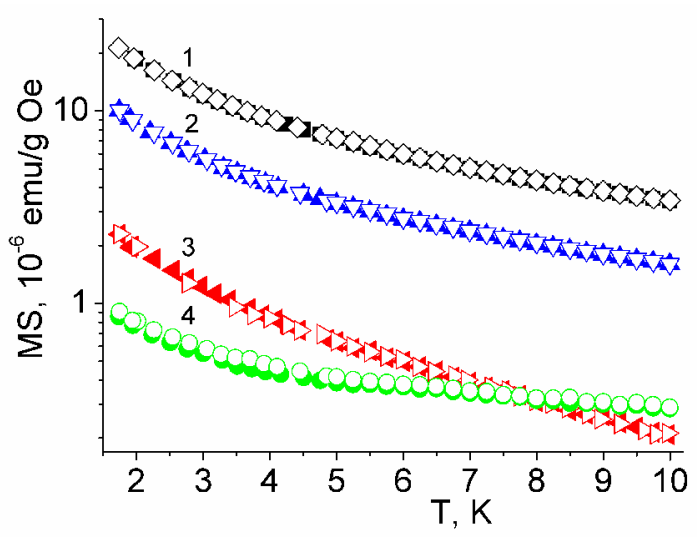

Fig. 2. MS vs. temperature at the magnetic field 300 Oe. The designations are the same as in Fig. 1.

(ii) If the distance along the surface from the ingot's beginning increases, the difference between magnetization of the samples in weak magnetic fields appears when the field changes up and down. The difference is larger at a lower intensity of the applied field and lower temperature of the sample (the samples 2 to 4$)$.

(iii) The magnitude of the mentioned divergences is the largest for the sample fabricated from the surface at the ingot's end (the sample 4). In this case, magnetization of the sample in the process of increasing the external magnetic field needs reduction of the field.

(iv) For the sample fabricated from the surface at the ingot end (the sample 4), there is also a special temperature dependence of MS. During a thermal cycle "heating-cooling" within the temperature range below $\sim 6 \mathrm{~K}$, the dependences $\chi(T)$ on heating and cooling diverge - MS on cooling is higher. Furthermore, within the temperature range above $\sim 4 \mathrm{~K}$, the sensitivity of the sample MS to temperature decreases sharply. As a result, at $T>8 \mathrm{~K}$, it becomes greater than the MS of the sample produced from the neighboring, the central area of the 
Table. The values of parameters used for calculation of magnetization of the investigated powder samples of PbTe:Eu.

\begin{tabular}{|c|c|c|c|c|c|c|}
\hline \multirow[t]{3}{*}{ Samples } & \multirow{3}{*}{$\begin{array}{l}\text { Position from the } \\
\text { ingot beginning } \\
\text { (total length } \\
\sim 30 \mathrm{~mm} \text { ), } \\
\mathrm{mm}\end{array}$} & \multicolumn{5}{|c|}{ Parameters } \\
\hline & & $\begin{array}{c}N_{\mathrm{Eu}} \\
\text { (singles), } \mathrm{cm}^{-3}\end{array}$ & $\begin{array}{c}N_{\mathrm{Eu}} \\
\text { (NN pairs), } \\
\mathrm{cm}^{-3}\end{array}$ & $\begin{array}{c}N_{\mathrm{Eu}} \\
\text { (NNN pairs), } \\
\mathrm{cm}^{-3}\end{array}$ & $\begin{array}{l}\chi_{\text {Matrix }}(0), \\
10^{-6} \mathrm{~cm}^{3} / \mathrm{g}\end{array}$ & \multirow[t]{2}{*}{$\begin{array}{c}\Delta \chi=\chi_{\exp }(10 \mathrm{~K})- \\
\chi_{c l}(10 \mathrm{~K}), \\
10^{-6} \mathrm{~cm}^{3} / \mathrm{g}\end{array}$} \\
\hline & & $\begin{array}{l}\% \text { singles from } \\
\text { the total of } \mathrm{Eu}\end{array}$ & \multicolumn{2}{|c|}{$\%$ pairs from the total of $\mathrm{Eu}$} & $\begin{array}{c}\text { For } \\
M(B)\end{array}$ & \\
\hline \multirow{2}{*}{1} & \multirow{2}{*}{$\begin{array}{c}\sim 0 \ldots 3 \\
\text { (the initial part of } \\
\text { the cone) }\end{array}$} & $2.46 \cdot 10^{19}$ & 0 & 0 & \multirow[t]{2}{*}{-0.41} & \multirow[t]{2}{*}{0} \\
\hline & & $100 \%$ & \multicolumn{2}{|c|}{$\mathbf{0 \%}$} & & \\
\hline \multirow[t]{2}{*}{2} & \multirow{2}{*}{$\begin{array}{c}\sim 3 \ldots 10 \\
\text { (lateral surface of } \\
\text { cone) }\end{array}$} & $1.0 \cdot 10^{19}$ & $1.8 \cdot 10^{18}$ & $7.6 \cdot 10^{17}$ & \multirow[t]{2}{*}{-0.41} & \multirow[t]{2}{*}{$\sim 0$} \\
\hline & & $\sim 80 \%$ & \multicolumn{2}{|c|}{$\sim 20 \%$} & & \\
\hline \multirow[t]{2}{*}{3} & \multirow{2}{*}{$\begin{array}{l}\sim 10 \ldots 20 \\
\text { (lateral surface of } \\
\text { cylinder) }\end{array}$} & $1.73 \cdot 10^{18}$ & $9.9 \cdot 10^{17}$ & $3.2 \cdot 10^{17}$ & \multirow[t]{2}{*}{-0.41} & \multirow[t]{2}{*}{$\sim+0.13$} \\
\hline & & $\sim 57 \%$ & \multicolumn{2}{|c|}{$\sim 43 \%$} & & \\
\hline \multirow[t]{2}{*}{4} & \multirow{2}{*}{$\begin{array}{l}\sim 20 \ldots 30 \\
\text { (lateral surface of } \\
\text { cylinder) }\end{array}$} & 0 & $1.04 \cdot 10^{18}$ & 0 & \multirow[t]{2}{*}{-0.41} & \multirow[t]{2}{*}{$\sim+0.52$} \\
\hline & & 0\% & \multicolumn{2}{|c|}{$100 \%$} & & \\
\hline \multirow[t]{2}{*}{ Pwd 1a [10] } & - & 0 & $2.0 \cdot 10^{18}$ & $2.7 \cdot 10^{17}$ & \multirow[t]{2}{*}{-0.44} & \multirow{2}{*}{$\begin{array}{c}\chi_{\text {Matrix }}(0) \text { for HT } \\
\chi(T)+0.93\end{array}$} \\
\hline & & 0\% & \multicolumn{2}{|c|}{$100 \%$} & & \\
\hline \multirow[t]{2}{*}{ Pwd $1 b[10]$} & - & 0 & $2.84 \cdot 10^{18}$ & $3.5 \cdot 10^{17}$ & \multirow[t]{2}{*}{-0.45} & \multirow{2}{*}{$\begin{array}{c}\chi_{\text {Matrix }}(0) \text { for HT } \\
\chi(T)+1.46\end{array}$} \\
\hline & & 0\% & \multicolumn{2}{|c|}{$100 \%$} & & \\
\hline
\end{tabular}

ingot (the sample 3), although, at the lowest investigated temperature $1.72 \mathrm{~K}$, MS of the sample 3 is more than 2.5 times higher than the MS of the sample 4.

\section{Discussion}

When studying the surface samples of PbTe:Eu crystals, two types of dependences were observed. The dependence of magnetization on magnetic field, diverging at a field close to $1 \mathrm{kOe}$ and lower under magnetic field changes up and down, as well as the dependence of MS on the specific temperature, decreasing sharply at a temperature only within a narrow temperature range below $\sim 4 \mathrm{~K}$ and practically independent of temperature at high temperatures. The dependences are explained by the influence of leadbased inclusions having the properties of type II superconductors at low temperatures [10]. Obviously, here we have the confirmation of the effect revealed in [10]. The present systematic study of the behavior of magnetization and MS of the surface layers of the $\mathrm{PbTe}: \mathrm{Eu}$ doped ingot provides information about the features of these inclusions formed during the crystal growth. They show that the probability of appearance of the lead-based inclusions passing into the superconducting state on cooling is different in different parts of the surface of the PbTe ingot grown from melt and increases towards the ingot's end. This result can be explained using the $\mathrm{PbTe}$ state diagram, the maxima of the solidus and liquidus curves of which are shifted towards the tellurium excess relative to the stoichiometric composition [11]. As a result, the beginning of the crystal ingot grown from melt is enriched with tellurium, and the end - with lead. Therefore, the probability of appearance of the leadbased inclusions increases towards the ingot's end.

The quantitative analysis of the obtained data provides additional information about surface properties, namely, that there are correlations in formation of the lead-based inclusions passing into the superconducting state during the cooling process and Eu magnetic-ordered centers within the surface layers of the PbTe:Eu ingot. The presence or absence of the correlations is implied from calculations of the magnetization and MS of the samples and their comparison with experimental data.

The sample magnetization $M$ was calculated as the sum of the following two components: magnetization of the undoped matrix $M_{\text {und }}$ and magnetization of the Eu impurity centers $M_{\mathrm{Eu}}$ :

$M=M_{\text {und }}+M_{\mathrm{Eu}}$.

According to $[12,13], M_{\mathrm{Eu}}$ was calculated as the sum of the contributions of single centers $M_{S}$, the nearest-neighbor (NN) pairs with ferromagnetic interaction $M_{P 1}$, and the next-nearest-neighbor (NNN) pairs $M_{P 2}$ with antiferromagnetic interaction: 
$M_{E u}=M_{S}+M_{P 1}+M_{P 2}$.

To calculate $M_{S}, M_{P 1}$, and $M_{P 2}$, the standard procedure and standard ratios $[12,14,15]$ were used. As the exchange integrals, we used their values for Eu ions in EuO normed by the lattice constant of PbTe: $J_{1} / k_{\mathrm{B}}=$ $0.056 \mathrm{~K}$ for ferromagnetic interaction of the $\mathrm{NN}$ pairs and $J_{1} / k_{\mathrm{B}}=-0.13 \mathrm{~K}$ for antiferromagnetic interaction of the NNN pairs [12].

At the given low temperature of investigation of the $M(H)$ dependences, the magnitude of $M_{\text {und }}$ was calculated as:

$M_{\text {und }}=\chi_{\text {Matrix }}(0) H$,

where $\chi_{\text {Matrix }}(0)$ is the MS of the undoped crystal matrix under condition $T \rightarrow 0 \mathrm{~K}$.

To calculate the temperature dependence of MS of the samples, the dependence $\chi_{\text {Matrix }}(T)$, according to [12], is considered as:

$$
\chi_{\text {Matrix }}(T)=\chi_{\text {Matrix }}(0)+2.5 \cdot 10^{-4} T-2.4 \cdot 10^{-7} T^{2} .
$$

The concentrations of $\mathrm{Eu}$ as a constituent part of different impurity centers (singles or pairs) and the magnitude of $\chi_{\text {Matrix }}(0)$ are considered as adjustable parameters for calculations. To fit the $M(H)$ dependences and determine the sample parameters, the magnetic field range in which the sample magnetizations agree well under changes of magnetic field up and down $(H \geq$ $4 \mathrm{kOe}$ ) was used.

The sample parameters for which the calculated $M(H)$ curves reproduce the experimental dependences in the best way are given in Table. It also provides with the data from [10]. Comparison of the calculated and experimental dependences $M(H)$ on the examples of the samples 1 and 4 , fabricated from the beginning and ending parts of the doped ingot, is shown in Figs 3 and 4.

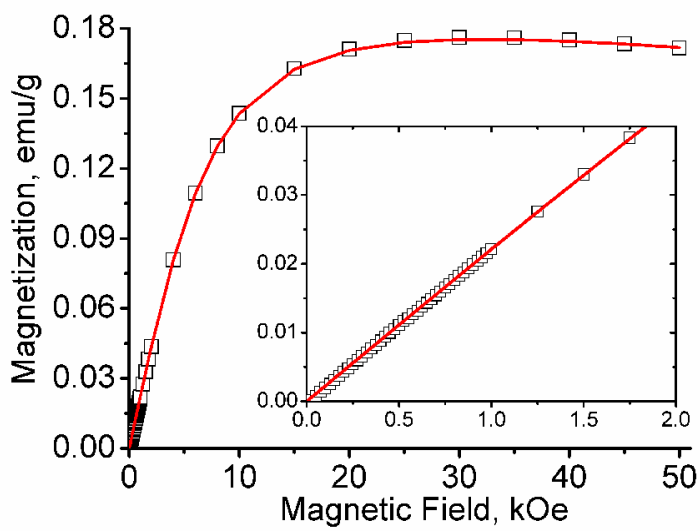

Fig. 3. Magnetization of the sample 1 vs. magnetic field $(T=$ $1.72 \mathrm{~K})$. The symbols are the experimental data; the solid lines are the calculated ones. Inset: the same at weak magnetic field.

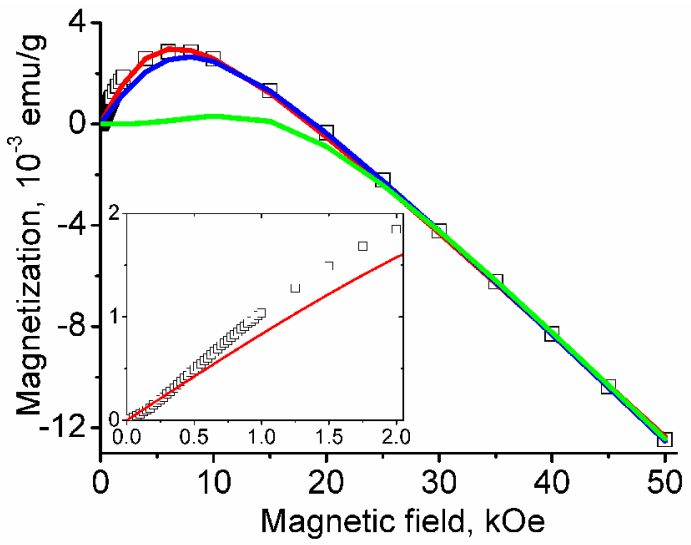

Fig. 4. Magnetization of the sample 4 vs. magnetic field $(T=1.72 \mathrm{~K})$. The symbols are the experimental data; the solid lines are the calculated ones for: the red line - $\mathrm{NN}$ pairs $\left(\chi_{\text {Matrix }}(0)=-0.41 \cdot 10^{-6} \mathrm{~cm}^{3} / \mathrm{g}, N_{\mathrm{Eu}}=-1.04 \cdot 10^{18} \mathrm{~cm}^{-3}\right)$; blue line - single $\mathrm{Eu}$ centers $\left(\chi_{\text {Matrix }}(0)=-0.426 \cdot 10^{-6} \mathrm{~cm}^{3} / \mathrm{g}, N_{\mathrm{Eu}}=\right.$ $\left.1.12 \cdot 10^{18} \mathrm{~cm}^{-3}\right)$; green line $-\mathrm{NNN}$ pairs $\left(\chi_{\text {Matrix }}(0)=\right.$ $\left.-0.435 \cdot 10^{-6} \mathrm{~cm}^{3} / \mathrm{g}, N_{\mathrm{Eu}}=1.2 \cdot 10^{18} \mathrm{~cm}^{-3}\right)$. Inset: the weak magnetic fields.

At least four results of the quantitative analysis of $M(H)$ dependences of the investigated samples deserve a special attention.

First, both single $\mathrm{Eu}$ centers and small $\mathrm{Eu}$ complexes are formed within the surface layers, but the ratio of their concentrations is different in different parts of the surface of the doped ingot and changes regularly along the surface (Table). At the beginning of the ingot, all the doping impurity is in a state of Eu single centers (the sample 1). This is clearly illustrated by comparison of the $M(H)$ dependences (Fig. 3) and temperature dependences of MS, which will be discussed below. As the distance from the ingot beginning increases, the small complexes of Eu impurity emerge within the ingot surface (the samples 2 and 3). Towards the end of the ingot, only small Eu-based complexes are formed within the surface layers (the sample 4). The falling section of the $M(H)$ dependence for this sample can be reproduced by combining the contributions of different Eu centers single ions, NN and NNN pairs, but within the whole range of magnetic fields, including the region of $M$ increase and maximum, the $M(H)$ dependence is described in the best way by the contribution of only NN Eu pairs with ferromagnetic interaction (Fig. 4).

Second, there is a correlation between formation of the Eu small magnetic complexes and the inclusions passing into the superconducting state on cooling within the surface of the PbTe:Eu doped ingot. In the sample 1, we observe neither any Eu complexes, nor any signs of magnetization, which can be related to formation of superconducting inclusions. These signs appear in the sample 2 and are clearer in the sample 3, where Eu emerges as a constituent of the complexes. And, finally, in the sample 4, where the whole Eu impurity is a 
constituent of the complexes, the presence of the superconducting inclusions is expressed especially clearly. These data are in very good agreement with the results of [10] also presented in Table, where these superconducting states were observed for the first time.

Third, the contribution from the small Eu-based complexes and crystal matrix reproduces the dependence $M(H)$ for different parts of the surface of the PbTe:Eu doped ingot differently. For the sample 1 fabricated from the ingot beginning, this reproduction is practically perfect within the entire range of the used magnetic fields from 0 to $50 \mathrm{kOe}$ (Fig. 3). For the sample 4 fabricated from the surface at the ingot end, the calculation almost perfectly reproduces the experiment only at relatively high magnetic fields $H \geq 4 \mathrm{kOe}$, and at weak fields from 0.5 to $2.0 \mathrm{kOe}$, the experimental values of $M$ are noticeably larger than the calculated ones (Fig. 4).

Fourth, a single value of the lattice MS $\chi_{\text {Matrix }}(0)=$ $=-0.41 \cdot 10^{-6} \mathrm{~cm}^{3} / \mathrm{g}$ (which is negative and is within the range of typical values for undoped $\mathrm{PbTe}$ [16]) describes very well the low temperature field dependences of magnetization of all the samples, but fails in the case of the MS temperature dependences. As seen from Fig. 5, only for the sample 1 the temperature dependence of MS is very well described by the same set of parameters as for the field dependence of magnetization (Fig. 3). A satisfactory convergence is also observed in the case of the sample 2. For the sample 3 and especially for the sample 4, the differences between calculations and experiments are very significant. When temperature increases from 1.7 to $10 \mathrm{~K}$, the difference between the experimental $\chi_{e x p}$ and calculated $\chi_{c l}$ values of MS $\Delta \chi=$ $\chi_{\exp }(10 \mathrm{~K})-\chi_{c l}(10 \mathrm{~K})$ increases to $+0.13 \cdot 10^{-6}$ and $+0.52 \cdot 10^{-6} \mathrm{~cm}^{3} / \mathrm{g}$, respectively. Even greater differences of this kind were observed earlier [10] for the similar PbTe:Eu surface samples, the contribution of the superconducting state to the properties of which was much larger (see Table).

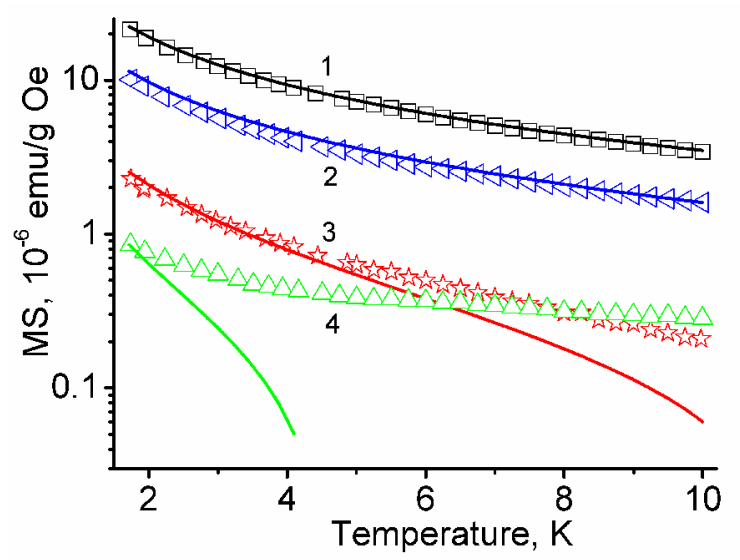

Fig. 5. MS of the investigated samples vs. temperature $(H=$ $300 \mathrm{Oe})$. The symbols are the experimental data; the solid lines are the calculated ones. The designations are the same as in Fig. 1.
So, the results of the theoretical analysis of the $M(H)$ and $\chi(T)$ dependences for the investigated samples allows us to state that within the surface layers towards the end of the ingot, some centers are formed, which strongly affect the magnetic properties of the surface, but these are absent at the beginning of the ingot. It is natural to assume that these centers are the mentioned above $\mathrm{Pb}$-based inclusions passing into the superconducting state on cooling. However, this assumption needs an explanation for at least two points. Firstly, how the $\mathrm{Pb}$-based inclusions can enhance magnetization of a sample due to the magnetic impurity of Eu in weak magnetic fields? Secondly, and the most important, why strong paramagnetism of the sample with $\mathrm{Pb}$-based inclusions disappears at low temperatures in strong magnetic fields, where it would be logical to expect destruction of the superconducting state of these inclusions (the critical magnetic field of $\mathrm{Pb}$ at $T \rightarrow 0 \mathrm{~K}$ $\left.H_{0}=803 \mathrm{Oe}\right)$ ?

If the discrepancy between the calculated and experimental magnitudes of the sample 4 magnetization at $T=1.72 \mathrm{~K}$ would be inverse to the obtained one that is if the experimental magnitude of $M$ was lower than the calculated one in weak magnetic fields, it could be explained by the impact of diamagnetism of some number of superconducting inclusions. However, transition of some inclusions into the superconducting state cannot cause any additional paramagnetism of the sample.

In principle, the presence of the inclusions passing into the superconducting state on cooling can enhance paramagnetism of $\mathrm{PbTe}: \mathrm{Eu}$ doped samples indirectly through amplification of exchange interaction between magnetic ions of Eu NN pairs and/or amplification of magnetic field acting on these pairs. If we assume that the ferromagnetic exchange interaction between $\mathrm{Eu}$ ions of NN pairs is amplified through the superconducting state, then $J_{1}$ should increase in the magnetic field range, where superconductivity exists, because the distribution of the Pb-based superconducting inclusions correlates with the distribution of Eu pairs. Increase of $J_{1}$ will lead to the increase of magnetization of $\mathrm{Eu} \mathrm{NN}$ pairs in the range of weak magnetic fields far from saturation. On the other hand, the investigated surface samples at temperatures below the critical one are in a mixed state, because they are a mixture of superconducting and normal phases. Under transition to the superconducting state, the superconducting inclusions push magnetic field out of their volume due to the Meissner effect. As a consequence, the local magnetic field outside the inclusions increases as compared to the applied one. Since the distribution of the $\mathrm{Eu} \mathrm{NN}$ pairs correlates with the distribution of the superconducting inclusions, the magnetic field acting on the Eu pairs increases and, therefore, magnetization of the sample increases, too. Both explanations allow to formally describe the experimental $M(H)$ dependence for the sample 4 at low temperatures. However, they cannot explain paramagnetism of the sample crystal matrix at high temperatures and simultaneously diamagnetism of the 
same matrix at low temperatures. It follows that these effects, even if they occur, will be only secondary. The primary effect should be the direct influence of the $\mathrm{Pb}$ based inclusions on the $M(H)$ and $\chi(T)$ dependences of the sample 4.

We suggest that this influence is realized through combined action of orientation and polarization constituents of the inclusion paramagnetism. The orientation constituent provides a stronger increase of the sample magnetization in weak magnetic fields. The polarization constituent is responsible for transition from the temperature-independent paramagnetic MS of the inclusion to its temperature-independent diamagnetic $\mathrm{MS}$, as the sample is cooled below the critical temperature $T_{c}$ of inclusion. The reasons for this assumption are listed below.

The $\mathrm{Pb}$-based inclusion can provide the necessary enhancement of the magnetization field dependence of the $\mathrm{PbTe}: \mathrm{Eu}$ doped sample in weak magnetic fields due to orientation paramagnetism, if they have an uncompensated spin. It follows from the fact that the Brillouin function determining magnetization of isolated paramagnetic centers $[14,15]$ saturates with the increase of applied magnetic field the faster, the smaller is the total spin $S_{o}$ of the magnetic center. Fig. 6 shows that all the $M(H)$ dependences of the sample 4 can be described very well if assuming that in addition to the crystal matrix and $\mathrm{Eu}$ ions its magnetization is determined also by the contribution of paramagnetic centers with the spin $1 / 2$. The magnetic-ordered Eu centers such as $\mathrm{NN}$ and $\mathrm{NNN}$ pairs in PbTe:Eu crystals, where $\mathrm{Eu}$ is a doping impurity, are formed mainly by europium and oxygen [12]. We found that the distribution of the Pb-based inclusions inside the surface layers of PbTe:Eu crystals correlated with the distribution of the small $\mathrm{Eu}$

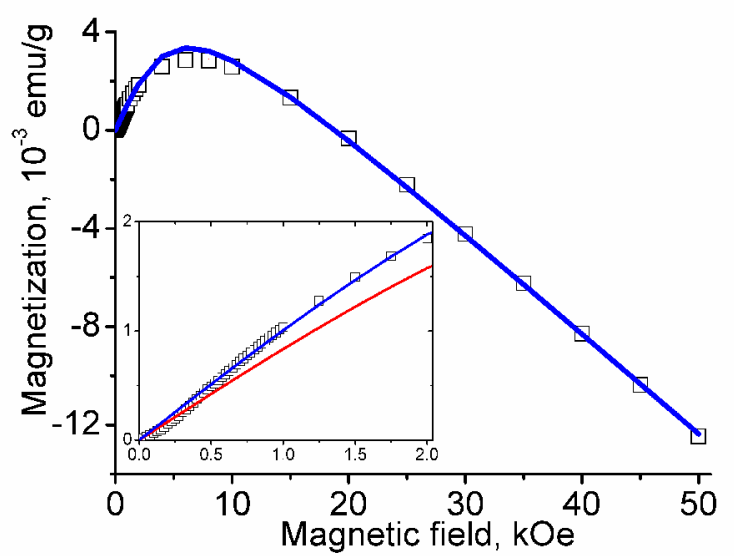

Fig. 6. Magnetization of the sample 4 vs. magnetic field $(T=$ $1.72 \mathrm{~K}$ ). The symbols are the experimental data; the solid line is the calculated ones for contributions of the $\mathrm{Eu} \mathrm{NN}$ pairs $\mathrm{S}_{o}\left(\mathrm{Eu}^{2+}\right)=7 / 2$, the paramagnetic single centers with $\mathrm{S}_{o}=1 / 2$, and the crystal matrix $\left(\chi_{\text {Matrix }}(0)=-0.41 \cdot 10^{-6} \mathrm{~cm}^{3} / \mathrm{g}\right)$. Inset: the weak magnetic fields. The red line shows the same dependence calculated for NN pairs as in Fig. 4. complexes. So, it is natural to assume that some portion of lead forming the $\mathrm{Pb}$-based inclusion may unite with oxygen, producing various oxides. This oxide, in particular, can be oxide $\mathrm{Pb}_{2} \mathrm{O}$, where lead is in the state of $\mathrm{Pb}^{+}$, and, therefore, has the uncompensated spin $1 / 2$ that is seven times smaller than the total spin of $\mathrm{Eu}^{2+}$ ions $-S_{o}\left(\mathrm{Eu}^{2+}\right)=7 / 2$. Then, the blue curve in Fig. 6 will correspond to the following concentrations of the paramagnetic centers under condition that $\chi_{\text {Matrix }}(0)=$ $-0.41 \cdot 10^{-6} \mathrm{~cm}^{3} / \mathrm{g}: N\left(\mathrm{Eu}^{2+}\right)=8.4 \cdot 10^{17} \mathrm{~cm}^{-3}$, and $N\left(\mathrm{~Pb}^{+}\right)=$ $4 \cdot 10^{18} \mathrm{~cm}^{-3}$.

The Pb-based inclusions can also be a source of paramagnetism due to the Van Vleck polarization constituent of MS. Van Vleck paramagnetism is known [17] to be a sum of terms $\left|\left\langle n\left|M_{z}^{(0)}\right| n^{\prime}\right\rangle\right|^{2} /\left(E_{n^{\prime}}^{(0)}-E_{n}^{(0)}\right)$, where $\left\langle n\left|M_{z}^{(0)}\right| n^{\prime}\right\rangle$ is a matrix element of the magnetic moment operator, $E_{n^{\prime}}^{(0)}-E_{n}^{(0)}$ is the energy difference between the normal $n$ and excited $n^{\prime}$ states of ions. Therefore, the following explanation of lattice paramagnetism of the sample 4 at elevated temperatures and its diamagnetism at $T=1.72 \mathrm{~K}$ can be suggested. If the temperatures are above $T_{c}$, the denominators $E_{n^{\prime}}^{(0)}-E_{n}^{(0)}$ are so small that the Van Vleck polarization constituent of MS of inclusion exceeds its Langevin diamagnetic constituent, the inclusion will give a positive addition to the MS of crystal lattice as a whole. As Van Vleck paramagnetism is almost independent of temperature, we obtain a significant weakening of the dependence of the total MS of the sample 4 on temperature relative to the other investigated samples. The energy of the ground state of inclusion $E_{n}^{(0)}$ should dramatically decrease under passing of the inclusion into the superconducting state. Therefore, at the temperatures lower than $T_{c}$, the ratio of the Van Vleck and Langevin constituents can be inversed due to the fact that the energy difference $E_{n^{\prime}}^{(0)}-E_{n}^{(0)}$ increases dramatically. Consequently, an additional paramagnetic contribution to MS of the crystal disappears. In order for the Van Vleck paramagnetic component of MS of inclusions at low temperatures to be suppressed throughout the investigated range of magnetic fields, it is necessary that for superconductivity of inclusions to be kept in this field range. It can happen only in the case when the top critical field $H_{c 2}$ of the $\mathrm{Pb}$-based inclusion is higher than $50 \mathrm{kOe}$. This situation is quite possible, given that the critical magnetic field $H_{c}$ of lead as I type superconductor is equal to $803 \mathrm{Oe}$ at $T \rightarrow 0 \mathrm{~K}$, and the top critical field $H_{c 2}$ in type II superconductors can by more than two orders of magnitude exceeds the critical field of I type superconductors, on base of which this type II superconductor is formed [18].

Finally, let's consider the reasons of correlations in formation of the small $\mathrm{Eu}$ complexes and $\mathrm{Pb}$-based 
inclusions within the surface layers of the $\mathrm{PbTe}: \mathrm{Eu}$ ingot, where the latter is grown from melt with such a low concentration of doping impurity that it is completely pushed out onto the surface of the ingot. They are caused by the properties of the PbTe state diagram and properties of segregation inherent to $\mathrm{Eu}$ impurity. As we noted above, the end part of the $\mathrm{PbTe}$ ingot growing from melt is enriched with lead because the maxima of the liquidus and solidus curves of the $\mathrm{PbTe}$ phase diagram are shifted towards the excess of tellurium relative to the stoichiometric composition. The surface of the PbTe:Eu ingot close to its end is enriched by europium and oxygen for two reasons. Due to the great value of the segregation coefficient of $\mathrm{Eu}$ in the case of its small concentrations in melt, $\mathrm{Eu}$ is always pushed out onto the lateral ingot's surface, which solidifies first. On the other hand, the inclusions of europium and oxygen, which are formed in front of the crystallization front, are pushed out of the solid to the liquid phase during solidification [9]. Since oxygen plays a crucial role in formation of small Eu complexes such as $\mathrm{NN}$ and NNN pairs in PbTe:Eu crystals [12], the lateral surface close to the end of PbTe:Eu ingot is enriched with these small complexes. Since in this part of the ingot surface, we practically see no isolated Eu centers, it may indicate that the amount of $\mathrm{Eu}$ ions is not sufficient to associate all the available oxygen in small complexes. The excess of oxygen can react with lead that is pushed out here. It promotes transformation of lead inclusions of type I superconductor (pure lead) into type II superconductor, which is lead doped with different impurities [18]. Hence, the observed correlation and specific influence of the $\mathrm{Pb}$ based inclusions on magnetic properties of the surface layers of PbTe:Eu doped crystals arise.

\section{Conclusions}

Magnetic properties of the surface layers in the $\mathrm{PbTe}: \mathrm{Eu}$ ingot grown using the Bridgman method from melt with the initial concentration of $\mathrm{Eu}$ impurity $N_{\mathrm{Eu}}^{\mathrm{int}}(m l)$ $1 \cdot 10^{19} \mathrm{~cm}^{-3}$ have been investigated. The measurements of magnetization and magnetic susceptibility within the temperature range 1.7 to $10.0 \mathrm{~K}$ and at magnetic field up to $50.0 \mathrm{kOe}$ were used for this purpose. Formation of various small units within the surface layers such as the single centers of $\mathrm{Eu}$, its $\mathrm{NN}$ pairs with ferromagnetic interaction, NNN pairs with antiferromagnetic interaction, as well as $\mathrm{Pb}$-based inclusions passing into the superconducting state on cooling, which determine magnetic properties of the layers, has been studied. The correlation between formation of the magnetic-ordered and superconducting surface states has been found. It consists in the fact that they both appear inside the surface layers of the ingot the more likely, the closer is a surface area to the end of the ingot. It has been shown that formation of the $\mathrm{Pb}$-based inclusions significantly enhances the field dependence of magnetization of the PbTe:Eu surface samples in weak magnetic fields, as well as dramatically influences magnetic susceptibility of their crystal matrix up to the inversion of its sign.

We suggest that the influence of the $\mathrm{Pb}$-based inclusions on magnetic susceptibility of crystal matrix of the PbTe:Eu surface samples is realized through combined action of the orientation and polarization paramagnetic constituents of the inclusions. The former are related to lead oxides as probable constituents of inclusions, the latter - to Van Vleck paramagnetism of the inclusions in general. A suitable oxide can be, for example, $\mathrm{Pb}_{2} \mathrm{O}$ oxide, where lead is in a state of $\mathrm{Pb}^{+}$, and, therefore, has the uncompensated spin $1 / 2$. The $\mathrm{Pb}$ based inclusions generally have to form type II superconductor with the top critical field higher than $50 \mathrm{kOe}$ as minimum, so that at low temperatures, the crystal matrix of the surface samples with inclusions can be diamagnetic as it follows from our experiments.

\section{References}

1. V.F. Masterov, Electronic structure of the rare earth impurities in III-V compounds // Fizika i Tekhnika Poluprovod. 27(9), p. 1435-1452 (1993), in Russian.

2. V.I. Mikityuk, D.M. Zayachuk, P.M. Starik, V.I. Garasim, V.P. Krasnodemskii, Effect of gadolinium on the properties of IV-VI crystals grown from the melt // Inorganic Materials, 31(10), p. 1197-1200 (1995).

3. F. Geist, W. Herbst, C. Mejía-García, H. Pascher, R. Rupprecht, Y. Ueta, G. Springholz, G. Bauer, and M. Tacke, Magneto-optical investigations of Eu-based diluted magnetic lead chalcogenides // Phys. Rev. B, 56(20), p. 13042-13053 (1997).

4. E.P. Skipetrov, N.A. Chernova, E.I. Slynko, Impurity-induced magnetism in $\mathrm{Pb}_{1-\mathrm{x}-\mathrm{y}} \mathrm{Ge}_{\mathrm{x}} \mathrm{Yb}_{\mathrm{y}} \mathrm{Te}$ // Phys. Rev. B, 66(8), 085204 (2002) (5 pages).

5. V. Jovovic, S.J. Thiagarajan, J. West, J.P. Heremans, T. Story, Z. Golacki, W. Paszkowicz, and V. Osinniy, Transport and magnetic properties of dilute rare-earth-PbSe alloys // J. Appl. Phys. 102, 043707 (2007).

6. A. Łusakowski, M. Górska, J.R. Anderson, Y. Dagan, Z. Gołacki, The magnetic contribution to the specific heat of $\mathrm{Pb}_{1-\mathrm{x}} \mathrm{Ge}_{\mathrm{x}} \mathrm{Te} / / \mathrm{J}$. Phys.: Condens. Matter, 21, 265802 (2009).

7. BM. Pinczolits, G. Springholz, and G. Bauer, Evolution of hexagonal lateral ordering in strainsymmetrized $\mathrm{PbSe} / \mathrm{Pb}_{1-\mathrm{x}} \mathrm{Eu}_{\mathrm{x}} \mathrm{Te}$ quantum-dot superlattices // Phys. Rev. B, 60(16), p. 1152411529 (1999)

8. E. Smajek, M. Szot, L. Kowalczyk et al., Optical and structural properties of $\mathrm{Pb}_{1-\mathrm{x}} \mathrm{Eu}_{\mathrm{x}} \mathrm{Te} / \mathrm{CdTe} / / \mathrm{G}$ aAs (001) heterostructures grown by MBE // J. Crystal Growth, 323, p. 140-143 (2011).

9. D.M. Zayachuk, O.S. Ilyina, A.V. Pashuk, V.I. Mikityuk, V.V. Shlemkevych, A. Csik, and D. Kaczorowski, Segregation of the Eu impurity as function of its concentration in the melt for 
growing of the lead telluride doped crystals by the Bridgman method // J. Crystal Growth, 376, p. 2834 (2013).

10. D.M. Zayachuk, V.I. Mikityuk, V.V. Shlemkevych, D. Kaczorowski, and O.S. Ilyina, Superconductivity of the surface layers of the doped PbTe:Eu crystals // Physica C, 483, p. 1-4 (2012).

11. G. Nimtz and B. Schlicht, Narrow-gap Semiconductors. Springer-Verlag, Berlin, 1985.

12. D.M. Zayachuk, V.I. Mikityuk, V.V. Shlemkevych, and D. Kaczorowski, Complex formation and background impurity of oxygen in the PbTe:Eu doped crystals grown from melt by the Bridgman method // J. Crystal Growth, 338, p. 35-41 (2012).

13. D.M. Zayachuk, V.I. Mikityuk, A.V. Pashuk, V.V. Shlemkevych, K.S. Ulyanitsky, D. Kaczorowski, Specific magnetic properties of the Eu-doped PbTe single crystals // J. Phys. Studies, 16(1/2), p. 1703-1-1703-7 (2012).
14. S. Isber, S. Charar, C. Fau, V. Mathet, M. Averous, and Z. Golacki, EPR spectra and magnetization of $\mathrm{Eu}^{2+}$ ions in PbSe // Phys. Rev. B, 52(3) p. 16781682 (1995).

15. M. Gorska, J.R. Anderson, C. Wolters, A. Lusakowski, T. Story, and Z. Golacki, Magnetization of $\mathrm{Sn}_{1-\mathrm{x}} \mathrm{Gd}_{\mathrm{x}} \mathrm{Te} / /$ Phys. Rev. B, 64(11), 115210 (2001) (8 pages).

16. D.M. Zayachuk, V.I. Mikityuk, P.M. Starik, Influence of intrinsic defects on the magnetic and optical properties of n-PbTe // Fizika i Tekhnika Poluprovod. 20(9), p. 1679-1684 (1986), in Russian.

17. S.V. Vonsovskii, Magnetism. Wiley, New York, 1974.

18. W. Buckel, Supraleitung: Grundlagen und Anwendungen. Weinheim/Bergstr:Physik Verlag $\mathrm{GmbH}, 1972$. 